\title{
Apendicitis en el paciente pediátrico: una revisión de la literatura
}

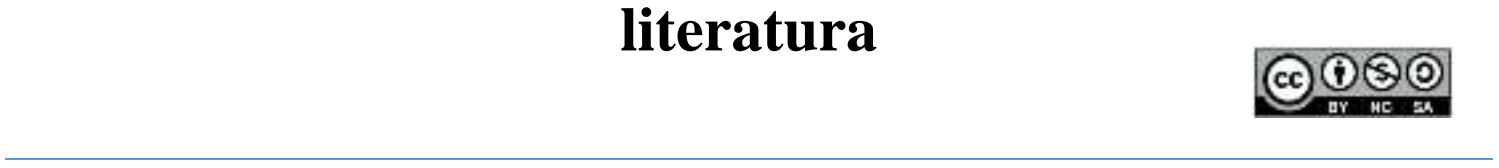

Appendicitis in the pediatric patient: a literature review

Gabriela Ivonne Cedeño López. ${ }^{1}$, Joamely Evelin Bermeo González. ${ }^{2}$, Lilibeth Kayra Espinoza Balseca. ${ }^{3}$, Johana Lizbeth Mora Sánchez. ${ }^{4} \&$ Adrián David Campoverde Cárdenas. ${ }^{5}$

DOI: https://doi.org/10.33262/anatomiadigital.v4i4.1947

\begin{abstract}
.
The expertise and knowledge of abdominal pain in pediatric patients is key when characterizing acute appendicitis. In our country Ecuador, it is one of the most frequent etiologies in morbidity rates, which depending on circumstances such as time, areas of difficult access, lack of health personnel, among others, there may be complications and fatal outcomes. The objective of presenting this review was to socialize the behavior of the pathology to medical students, physicians and

\section{Resumen.}

La experticia y conocimiento sobre dolor abdominal en pacientes pediátricos es clave al momento de caracterizar una apendicitis aguda. En nuestro país Ecuador, es una de las etiologías más frecuentes en tasas de morbilidad, dependiendo de las circunstancias como tiempo, áreas de difícil acceso, falta de personal de salud, entre otras, puede existir complicaciones y fatales desenlaces. El objetivo de la presentación de esta revisión fue socializar el comportamiento de la

\footnotetext{
${ }^{1}$ Centro de Salud Tipo C Recreo 2. Guayaquil, Ecuador.

${ }^{2}$ Interhospital, Auditoría Médica, Guayaquil, Ecuador. joamelyta12@hotamil.com

${ }^{3}$ Distrito 09D24 Durán, Samborondón Salud, Guayaquil, Ecuador. liilita_15@ hotmail.com

${ }^{4}$ Distrito 09D24 Durán, Samborondón Salud, Guayaquil Ecuador. joha_lizz91@ @otmail.com

5 Instituto Ecuatoriano de Seguridad Social, Centro de Salud Tipo B, El Oro, Ecuador. adccardenas@icloud.com
} 
health personnel in general, who are in direct care of patients with abdominal pain. The highlights of this disease have been clearly and precisely written, from its origin to its treatment in pediatric patients. The purpose of presenting this review is to provide the key characteristics of acute appendicitis for effective diagnosis and treatment.

Keywords: appendicitis, pediatrics, ultrasonography, signs and symptoms, abdominal pain. patología a estudiantes de medicina, médicos y personal de salud en general, quienes están en atención directa del paciente con dolor abdominal. Se ha redactado de forma clara y precisa lo más destacado de esta enfermedad, desde su origen hasta su tratamiento en pacientes pediátricos. La finalidad de la presentación de esta revisión es aportar con las características claves de la apendicitis aguda para un diagnóstico eficaz y su terapéutica.

Palabras claves: apendicitis, pediatría, ultrasonografía, signos y síntomas, dolor abdominal.

\section{Introducción}

La primera descripción formal del apéndice fue realizada por Berengario DaCapri en 1521, posteriormente Andrea Vesalio en su gran trabajo "De Humani Corporis Fabrica" publicado en 1523 realiza una detallada descripción de dicho órgano. Existe evidencia sobre la inflamación del apéndice que data de la era Bizantina, incluso se hallaron momias egipcias con adherencias en el cuadrante inferior derecho sugerentes de apendicitis. En 1544 se realiza la primera descripción del cuadro clínico de la patología por parte de Jean Fernel, mientras que Lorenz Heister describió en 1711 el apéndice perforado con absceso (Williams, 1983).

La primera apendicectomía realizada en la historia se le atribuye a Claudius Amyand quien en 1735 reportó el primer caso exitoso de extirpación del apéndice vermiforme en un niño de 11 años1. Posterior a este primer logro quirúrgico se describe la técnica para laparotomía utilizada clásicamente en 1894 por Charles McBurney (McBurney, 1891; 1894).

El dolor abdominal es uno de los motivos de consulta más comunes en la sala de emergencias, siendo la apendicitis la emergencia quirúrgica más frecuente en el paciente pediátrico, se realizan aproximadamente 70000 apendicectomías en pacientes pediátricos con un costo aproximado de $\$ 9000$ por paciente en los EE. UU. cada año (Healthcare Cost and Utilization Project [HCUP], 2019).

En el Ecuador, la apendicitis aguda fue la primera causa de morbilidad en pacientes entre 12 a 17 años en el año 2019, con 6.256 egresos reportados (Instituto Nacional de Estadísticas y Censos [INEC], 2019). 
El objetivo de esta revisión fue presentar datos característicos de esta patología, con la finalidad de difundir esta información a todo el personal de salud quien evalúa todos los días pacientes con dolor abdominal.

\section{Epidemiología}

Los casos de apendicitis van de 74 a 96 por cada 100 mil individuos con una media de 85 , de los cuales 65 son apéndices no perforadas y cerca de 20 perforadas, esto quiere decir un tercio de los casos se presentan con una perforación del apéndice, de hecho el riesgo de perforación aumenta cuanto menor es el niño, aumentando la incidencia de perforación hasta un $82 \%$ en menores de 5 años y casi un $100 \%$ en menores de un año (Körner et al., 1997; Coward et al., 2016; Ferris et al., 2017; Marzuillo, 2015; Nance et al., 2000).

La incidencia a través de los años ha variado, desde los inicios del siglo 20 hasta los inicios del presente milenio pasando de más de 150 casos por cada 100 mil a menos de 80 por cada de 100 mil habitantes (Ferris et al., 2017). La mayoría de los casos se presentan en adolescentes y adultos jóvenes de 13 a 40 años (Körner et al., 1997 \& Coward et al., 2016), es ligeramente más prevalente en hombres que en mujeres $(60 \%$ vs 40\%), además el riesgo aumenta con las diferencias étnicas, como lo es en hispanos, asiáticos y nativos americanos, en nativo africanos el riesgo disminuye (Marzuillo, 2015; Nance et al., 2000).

En el Ecuador, la apendicitis aguda es la causa principal de cirugías de emergencia pediátrica, las tasas de complicación se encuentran entre 20 al 70\%, según reportes a nivel mundial y es más elevada con el 80 al $100 \%$ en pacientes pediátricos menores de 3 años (Ascanio, 2021).

Los casos de apendicitis aguda se redujeron durante la pandemia, hasta en un 50\%, con un incremento de los casos complicados (gangrena o perforación), hasta en el $90 \%$. Se constató un incremento del uso de tratamiento conservador hasta en el 88,9\% de los casos; sin embargo, la apendicectomía laparoscópica sigue siendo el tratamiento más utilizado. La epidemiología y abordaje terapéutico de la apendicitis aguda se ha modificado con la pandemia por COVID-19; con un incremento de casos complicados y el incremento del uso de tratamiento conservador (Cárdenas, 2021).

\section{Fisiopatología}

Existen diferencias significativas entra la anatomía del apéndice del neonato y del adulto, en primer lugar el apéndice del neonato apenas mide $4,5 \mathrm{~cm}$, mientras que el del adulto alcanza los 9,5cm (Buschard \& Kjaeldgaard, 1973), ciertas características como el hecho de que el apéndice tenga forma de embudo, se encuentre la mayor parte de tiempo en decúbito supino, tenga una dieta líquida y baja frecuencia de infecciones de tracto gastrointestinal hacen que sea raro el cuadro de apendicitis aguda en neonatos (Collins, 1973). Existe evidencia de que la lactancia materna reduce el riesgo de padecer apendicitis (Alves et al., 2008). 
Los lactantes mayores (1 a 2 años), presentan una anatomía con respecto al apéndice muy similar a la de los adultos, conformen crecen se presenta una hiperplasia folicular que alcanza su máximo en la adolescencia, período de máxima incidencia de apendicitis (Addiss et al., 1990). Los niños pequeños poseen un omento poco desarrollado incapaz de limitar la efusión purulenta producida por la perforación del apéndice, aumentando así la probabilidad de producir una peritonitis secundaria a la perforación, y a su vez se explica la baja incidencia de abscesos localizados en niños pequeños (Gilbert et al., 1985).

\section{Manifestaciones clínicas}

La sintomatología es muy variada, mientras más pequeño es el niño, más inespecífica es, para los niños escolares es más fácil describir los síntomas, el dolor es el síntoma más prevalente en niños menores de 5 años con una prevalencia de hasta el $94 \%$, seguido de vómito en un $83 \%$, fiebre en un $80 \%$ e inapetencia en un $70 \%$ (Bundy et al., 2002). Los signos varían dependiendo del cuadro fisiopatológico, aquellos que tienen una apendicitis imperforada el signo más prevalente será la sensibilidad en el cuadrante inferior derecho hasta con un 63\% de prevalencia, mientras que para los apéndices perforados la defensa abdominal (Signo de Blumberg) se presenta hasta en un $79 \%$ (Nance et al., 2000).

\section{Diagnóstico}

En 1986 fue publicado por Alvarado la herramienta de evaluación clínica de apendicitis en adultos con los criterios del MANTRELS (véase tabla 1), escala que ha tenido muy buena acogida mundialmente por haber demostrado una sensibilidad del $89 \%$ y una especificidad del 80 \% (Ohle et al., 2011; Alvarado, 1986). En 1997 MacKlin intentó validar la escala para pacientes pediátricos, obteniendo una sensibilidad y especificidad menor al 80\% (Macklin et al., 1997).

El ultrasonido a pesar de ser un método dependiente del operador ha demostrado ser el estudio de imagen más útil en pacientes pediátricos, además de tener la ventaja adicional de que no irradia a los pacientes, su sensibilidad va de un 89\% a 94\% (Weyant et al., 2003; Blebea et al., 2003).

\section{Tabla 1}

Mnemotecnia MANTRELS para el diagnóstico de apendicitis aguda

\begin{tabular}{lll}
\hline Symptoms & Migration & 1 \\
& Anorexia-acetone & 1 \\
\multirow{3}{*}{ Signs } & Nausea-vomiting & 1 \\
& Tenderness in in right lower quadrant & 2 \\
& Rebound pain & 1 \\
\multirow{2}{*}{ Laboratory } & Elevation of temperature & 1 \\
& Leukocytosis & 2 \\
& Shift to the left & 1 \\
\hline
\end{tabular}

Fuente: Tomado de Alvarado (1986). A practical score for the early diagnosis of acute appendicitis 


\section{Tratamiento}

La intervención quirúrgica laparoscópica es de elección para resolución del cuadro frente a una apendicitis no perforada, no existen diferencias entre el tipo de abordaje, ya sea que se realice una incisión o tres, con respecto a infecciones del sitio quirúrgico, dosis de analgésicos, tiempo de recuperación y estadía hospitalaria (St. Peter et al., 2011).

Para el manejo de la apendicitis perforada, se requiere además de la intervención quirúrgica la utilización de antibióticos en el postoperatorio hasta la resolución clínica del cuadro, la terapia antibiótica utilizada es una combinación triple de ampicilina, gentamicina y clindamicina (Results Of the North American trial, 1994; Nadler et al., 2003), la piperacilina/tazobactam ha resultado igual de eficaz que la terapia múltiple (Nadler et al., 2003), a su vez la combinación ceftriaxona más metronidazol en dosis única diaria ha demostrado ser igual de eficaz que las anteriores combinaciones y menos costosa (St. Peter et al., 2008).

\section{Conclusiones}

- El conocimiento de la historia, la epidemiología de la apendicitis aguda es muy importante, así como su fisiopatología, características de presentación clínica con la finalidad de brindar un diagnóstico oportuno y un tratamiento adecuado y eficaz a este tipo de patología en los pacientes pediátricos, reduciendo así tasas de complicaciones a corto, mediano y largo plazo en nuestro país.

- La apendicitis aguda es una patología con características clínicas ya descritas desde hace muchos años, debido a esto, la mayoría de las referencias bibliográficas pertenecen a tiempos memorables.

\section{Referencias bibliográficas}

Addiss, D.G., Shaffer, N., Fowler B.S., Tauxe R.V. (1990). The epidemiology of appendicitis and appendectomy in the United States. Am J Epidemial 132: 910925 [PMID: 2239906]. https://pubmed.ncbi.nlm.nih.gov/2239906-theepidemiology-of-appendicitis-and-appendectomy-in-the-united-states/

Alvarado, A. (1986). A practical score for the early diagnosis of acute appendicitis. Ann Emerge Med. 15(5):557-64. https://pubmed.ncbi.nlm.nih.gov/3963537-apractical-score-for-the-early-diagnosis-of-acute-appendicitis/

Alves, J.G., Figueiroa, J.N., Barros, I. (2008). Does breast feeding provide protection against acute appendicitis? A case-control study. Trop Doct 38: 235-236 [PMID: $18820196 \quad$ DOI: $10.1258 /$ td.2008.070404]. https://pubmed.ncbi.nlm.nih.gov/18820196-does-breast-feeding-provideprotection-against-acute-appendicitis-a-case-control-study/

Ascanio, W. (2021). Comparación de antibióticoterapia en la apendicitis complicada en pediatría. Hospital de Especialidades Carlos Andrade Marín, Unidad de Cirugía 
Pediátrica. Quito-

Ecuador. https://revistahcam.iess.gob.ec/index.php/cambios/article/view/585/456

Blebea, J.S., Meilstrup, J.W., Wise, S.W. (2003). Appendiceal imaging: Which test is best? Semin Ultrasound CT MR 24:91-5. https://pubmed.ncbi.nlm.nih.gov/12744501-appendiceal-imaging-which-test-is-best/

Bundy, D.G., Byerley, J.S., Liles, E.A., Perrin, E.M., Katznelson, J., Rice, H.E. (2002). Does this child have appendicitis? JAMA 298: 438-451 [PMID: 17652298 DOI: 10.1001/jama.298.4.438].

https://www.ncbi.nlm.nih.gov/pmc/articles/PMC2703737/

Buschard, K. \& Kjaeldgaard, A. (1973) Investigation and analysis of the position, fixation, length and embryology of the vermiform appendix. Acta Chir Scand 139: 293-298 [PMID: 4698491]. https://pubmed.ncbi.nlm.nih.gov/4698491investigation-and-analysis-of-the-position-fixation-length-and-embryology-ofthe-vermiform-appendix/

Cárdenas Reyes, D. A. (2021). Incidencia de apendicitis aguda en el contexto de la pandemia por covid-19. Revisión sistemática. [Trabajo de Titulación modalidad Artículo Profesional de Alto Nivel presentado como requisito previo para optar por el Título de Especialista en Cirugía General]. UCE.

Collins, D.C. (1973). 71,000 human appendix specimens a final report, summarizing forty years' study. Am J Proctol 14: 265-281 [PMID: 14098730]. https://pubmed.ncbi.nlm.nih.gov/14098730-71000-human-appendix-specimensa-final-report-summarizing-forty-years-study/

Coward, S., Kareemi, H., Clement, F., Zimmer, S., Dixon, E., Ball, C.G. (2016) Incidence of appendicitis over time: A comparative analysis of an administrative healthcare database and a pathology-proven appendicitis registry. PLoS One. 11(11):1-12. https://pubmed.ncbi.nlm.nih.gov/27820826/

Ferris, M., Quan, S., Kaplan, B.S., Molodecky, N., Ball, C.G., Chernoff, G.W. (2017) The Global Incidence of Appendicitis. Ann Surg. 266(2):237-41. https://pubmed.ncbi.nlm.nih.gov/28288060-the-global-incidence-ofappendicitis-a-systematic-review-of-population-based-studies/

Gilbert, S.R., Emmens, R.W., Putnam, T.C. (1985). Appendicitis in children. Surg Gynecology Obstet 161: 261-265 [PMID: 4035541]. https://europepmc.org/article/med/4035541

Healthcare Cost and Utilization Project [HCUP]. (2019) The SAGE encyclopedia of pharmacology and society. SAGE Publications. http://sk.sagepub.com/reference/the-sage-encyclopedia-of-pharmacology-andsociety/i5903.xml 
Instituto Nacional de Estadísticas y Censos [INEC]. (2019). Registro de Estadísticas de Camas $\quad \mathrm{y} \quad$ Egresos Hospitalarios. https://www.ecuadorencifras.gob.ec/documentos/webinec/Estadisticas_Sociales/Camas_Egresos_Hospitalarios/Cam_Egre_Hos_2019/ Boletin\%20tecnico\%20ECEH_2019.pdf

Körner, H., Söndenaa, K., Söreide, J.A., Andersen, E., Nysted, A., Lende, T.H. (1997) Incidence of acute nonperforated and perforated appendicitis: Age- specific and sex-specific analysis. World J Surg. 21(3):313-7. https://pubmed.ncbi.nlm.nih.gov/9015177-incidence-of-acute-nonperforatedand-perforated-appendicitis-age-specific-and-sex-specific-analysis/

Macklin, C.P., Radcliffe, G.S., Merei, J.M. (1997). A prospective evaluation of the modified Alvarado score for acute appendicitis in children. Ann R Coll Surg Engl 79:203-5. https://pubmed.ncbi.nlm.nih.gov/9196342-a-prospectiveevaluation-of-the-modified-alvarado-score-for-acute-appendicitis-in-children/

Marzuillo, P. (2015). Appendicitis in children less than five years old: A challenge for the general practitioner. World J Clin Pediatr. 4(2):19. https://www.ncbi.nlm.nih.gov/pmc/articles/PMC4438437/

McBurney, C. (1891) The indications for Early laparotomy in appendicitis. N Y State Med J. 234:254. https://www.ncbi.nlm.nih.gov/pmc/articles/PMC1428594/

McBurney, C. (1894) The Incision Made in the Abdominal Wall in Cases of Appendicitis, with a Description of a New Method of Operating. N Y State Med J. 38:43. https://www.ncbi.nIm.nih.gov/pmc/articles/PMC1493708/

Nadler, E.P., Reblock, K.K., Ford, H.R. (2003). Monotherapy versus multidrug therapy for the treatment of perforated appendicitis in children. Surg Infect (Larchmt). 4:327-333. https://www.ncbi.nlm.nih.gov/pmc/articles/PMC3082440/

Nance, M.L., Adamson, W.T., Hedrick, H.L. (2000) Appendicitis in the young child: a continuing diagnostic challenge. Pediatr Emerge Care. 16:160-162. https://pubmed.ncbi.nlm.nih.gov/10888451-appendicitis-in-the-young-child-acontinuing-diagnostic-challenge/

Ohle, R., O’Reilly, F., O’Brien, K.K., Fahey, T., Dimitrov, B.D. (2011). The Alvarado score for predicting acute appendicitis: A systematic review. BMC Med. 9. Available in: https://pubmed.ncbi.nlm.nih.gov/22204638-the-alvarado-score-forpredicting-acute-appendicitis-a-systematic-review/

Results of the North American trial of piperacillin/tazobactam compared with clindamycin and gentamicin in the treatment of severe intra-abdominal infections. (1994). Investigators of the Piperacillin/Tazobactam Intra-abdominal Infection Study Group. Eur J Surg Suppl.573:61-66. https://pubmed.ncbi.nlm.nih.gov/7524798-results-of-the-north-american-trial-of- 
piperacillintazobactam-compared-with-clindamycinandgentamicin-in-thetreatment-of-severe-intra-abdominal-infections-investigators-of-thepiperacillintazobactam-intra-abdominal-infection-study-group/

St. Peter, S.D., Tsao, K., Spilde, T.L. (2008). Single daily dosing ceftriaxone and metronidazole vs standard triple antibiotic regimen for perforated appendicitis in children: a prospective randomized trial. J Pediatr Surg. 43:981-985. Available in: https://pubmed.ncbi.nlm.nih.gov/18558169-single-daily-dosing-ceftriaxoneand-metronidazole-vs-standard-triple-antibiotic-regimen-for-perforatedappendicitis-in-children-a-prospective-randomizedtrial/?from_single_result=Single+daily+dosing+ceftriaxone+and+metronidazole + vs+standard+triple+antibiotic+regimen+for+perforated+appendicitis+in+childr en $\% 3 \mathrm{~A}+\mathrm{a}+$ prospective+randomized+trial

St. Peter, S.D., Adibe, O.O., Juang, D. (2011). Single incision versus standard 3-port laparoscopic appendectomy: a prospective randomized trial. Ann Surg. 254:586590. Available in: https://pubmed.ncbi.nlm.nih.gov/21946218-single-incisionversus-standard-3-port-laparoscopic-appendectomy-a-prospective-randomizedtrial/

Weyant, M.J., Eachempati, S.R., Maluccio, M.A. (2003). Is imaging necessary for the diagnosis of acute appendicitis? Adv Surg 37:327-45. https://pubmed.ncbi.nlm.nih.gov/12953640-is-imaging-necessary-for-thediagnosis-of-

acuteappendicitis/?from_term=Is+imaging+necessary+for+the+diagnosis+of+ac ute+appendicitis\%3F\&from_filter=pubt.review

Williams, R.G. (1983) Presidential address: a history of appendicitis. Ann Surg. 1983; 197:495-506. Available in: https://pubmed.ncbi.nlm.nih.gov/6342553presidential-address-a-history-of-appendicitis-with-anecdotes-illustrating-itsimportance/

\section{【Liencia}




\section{PARA CITAR EL ARTÍCULO INDEXADO.}

Cedeño López, G. I., Bermeo González, J. E., Espinoza Balseca, L. K., Mora Sánchez, J. L., \& Campoverde Cárdenas, A. D. (2021). Apendicitis en el paciente pediátrico: una revisión de la literatura . Anatomía Digital, 4(4), 150-158. https://doi.org/10.33262/anatomiadigital.v4i4.1947

\section{LCiencia}

El artículo que se publica es de exclusiva responsabilidad de los autores y no necesariamente reflejan el pensamiento de la Revista Anatomía Digital.

El artículo queda en propiedad de la revista y, por tanto, su publicación parcial y/o total en otro medio tiene que ser autorizado por el director de la Revista Anatomía Digital.
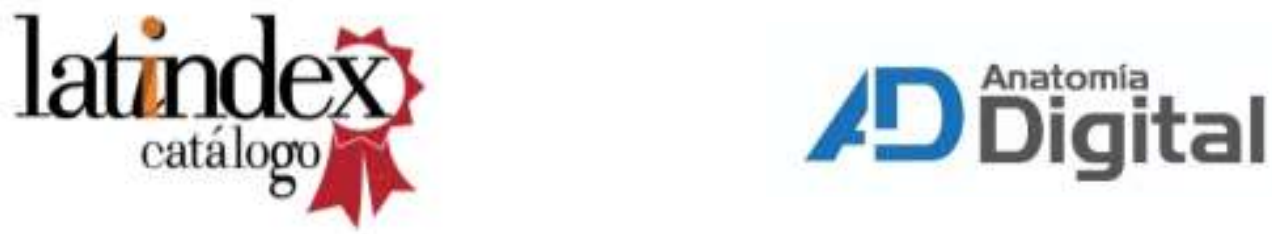